\title{
Determination of antibiotic resistance and minimum inhibitory concentration of meropenem and imipenem growth in Klebsiella strains isolated from urinary tract infection in Shahrekord educational hospitals
}

\author{
Farshad Kakian $^{1}{ }^{\mathbb{D}}$, Behnam Zamzad $^{2 *}$, Abolfazl Gholipour ${ }^{3}$, Kiarash Zamanzad $^{4}$ \\ ${ }^{1}$ Ph.D. Student of Medical Bacteriology, Shiraz University of Medical Sciences, Faculty of Medicine, Department of \\ Bacteriology, Shiraz, Iran \\ ${ }^{2}$ Department of Medical Bacteriology, Shahrekord University of Medical Sciences, Shahrekord, Iran \\ ${ }^{3}$ Ph.D., Department of Medical Bacteriology, Shahrekord University of Medical Sciences, Shahrekord, Iran \\ ${ }^{4}$ Student of Veterinary Medicine, University of Shahrekord, Shahrekord, Iran
}

*Corresponding Author: Behnam Zamzad, Professor, Cellular and Molecular Research Center, Shahrekord University of Medical Sciences, Shahrekord, Iran. Tel: +989131815136, Email: bzamanzad@yahoo.com

\begin{abstract}
Background and aims: Klebsiella is an opportunistic organism that is the cause of severe diseases such as pneumonia, septicemia, and urinary tract infections (UTIs). In addition, high antibiotic resistance has challenged the treatment of this bacterium. However, carbapenem antibiotics are considered as the therapeutic agents for selecting the treatment of penicillin- and cephalosporin-resistant gram-negative bacterial infections. The present study aimed to determine the resistance and minimum inhibitory concentration (MIC) of meropenem and imipenem.

Methods: A total of 80 Klebsiella spp isolated from UTIs were collected in various educational wards (i.e., urology, obstetrics, and gynecology, as well as the units of infectious diseases, internal medicine, and intensive care) in different hospitals of Shahrekord. The isolates were then identified by using biochemical tests. Further, disc diffusion method was employed to determine the antibiotic resistance. Furthermore, MIC was estimated by the Epsilon-test strip. Moreover, $\mathrm{P}=\mathrm{Q}=0.50$, an error of 0.05 , and an accuracy of 0.11 were considered for determining the sample size $(n=80)$.

Results: Based on the results of disc diffusion method, 24 strains were resistant to meropenem and imipenem. Additionally, the MIC was 24 (30\%) by the E-test. In addition, 24 isolates had a MIC of $\geq 4 \mu \mathrm{g} / \mathrm{mL}$ for meropenem and imipenem and thus were resistant while 18 isolates were found to have a MIC of $1 \leq \mathrm{MIC}<4 \mu \mathrm{g} / \mathrm{mL}$ and therefore, were considered semi-sensitive $(P<0.001)$.

Conclusion: In general, Klebsiella strains were found to be resistant to meropenem and imipenem. Therefore, rapid and accurate identification of these strains and the selection of appropriate antibiotics can help quickly eradicate the infections caused by these bacteria. Accordingly, a waste of time, the consumption of medication, or even an increased resistance are prevented.

Keywords: Klebsiella, Antibiotic resistance, Meropenem, Imipenem
\end{abstract}

Received: 14 November 2017, Accepted: 15 September 2018, ePublished: 4 April 2019

\section{Introduction}

Urinary tract infection (UTI) is the second leading reported infection in patients since nearly seven million patients are annually treated by the doctors (1). The presence of over $10^{5}$ colony forming units $(\mathrm{CFU}) / \mathrm{mL}$ of a bacterium in the urine sample indicates the UTI. Klebsiella strains are one of the major bacterial causes of the UTI (2). Klebsiella is an opportunistic bacterium which is related to severe conditions such as pneumonia, septicemia, and UTIs. The importance of this bacterium, as a human pathogen, is associated with the development of hospital infections in patients with immunodeficiency and underlying diseases such as diabetes mellitus and chronic pulmonary problems (3). UTIs are the most common infections among Klebsiella infections. The risk of UTI acquisition is far higher than the normal rate in people with bladder infections and diabetes (4). In recent years, the emergence of Klebsiella strains with multidrug resistance (MDR) has been observed due to the overthe-counter and excessive consumption of the antibiotics (5). In addition, antimicrobial resistance is always a major concern for human health and affects patients in hospitals across the world (6,7). Further, carbapenem antibiotics include ertapenem, imipenem, doripenem, and meropenem. The carbapenemase enzymes belong to the molecular classes $\mathrm{A}, \mathrm{B}$, and $\mathrm{D}$, which are able to inactivate carbapenem antibiotics such as meropenem and imipenem. Among the carbapenemase enzymes,

(c) 2019 The Author(s); Published by Shahrekord University of Medical Sciences. This is an open-access article distributed under the terms of the Creative Commons Attribution License (http://creativecommons.org/licenses/by/4.0), which permits unrestricted use, distribution, and reproduction in any medium, provided the original work is properly cited. 
Klebsiella pneumoniae carbapenemase, which is a class A carbapenemase, is inhibited by different degrees of clavulanate and hydrolyzes penicillins and cephalosporins more than carbapenems. This enzyme is considered an extended broad-spectrum $\beta$-lactamase (ESBL) since it lacks a robust carbapenemase activity. Furthermore, class B carbapenemases (Bush group 3) are known as the metallo-beta-lactamases that hydrolyze carbapenem antibiotics except for the aztreonam and are resistant to $\beta$-lactamase enzyme inhibitors such as clavulanate. However, these lactamases are inhibited by the chelating agents such as ethylenediaminetetraacetic acid which can degrade and inhibit carbapnemase enzymes, and therefore, it is a component of the diagnostic tests for these enzymes $(8,9)$. Carbapenem antibiotics are the drugs of the choice for treating the infections caused by gram-negative bacilli resistant to penicillin and cephalosporins due to being a broad spectrum and lacking the hydrolysis by beta-lactams $(10,11)$. Moreover, these antibiotics are currently regarded as the drugs of choice for treating serious infections related to MDR and ESBL-producing Enterobacteriaceae $(12,13)$. Infections caused by the MDR gram-negative bacteria lead to higher morbidity and mortality, longterm hospital stay, increased health care costs, and limited therapeutic options $(14,15)$. Therefore, these resistant bacteria should be identified using different methods such as antibiotic susceptibility testing, the determination of minimum inhibitory concentration (MIC), and genotype confirmatory tests including a polymerase chain reaction. Epsilon-test (E-test) is the most accurate method for determining the MIC of the antibiotics (16). If the bacterium is resistant to carbapenems, treatment with carbapenem alone cannot lead to patient recovery while it leads to the emergence of antibiotic-resistant bacteria, prolong medical treatments, or even mortality in the patients. Accordingly, the current study sought to detect meropenem- and imipenem-resistant Klebsiella strains by disc diffusion method in patients with UTI and to evaluate the resistance by MIC of these strains using the E-test strip.

\section{Materials and Methods}

In this descriptive-analytical study, 80 Klebsiella isolates (i.e., 74 pneumonia and 6 oxytoca isolates) were collected from patients with UTI in different wards (e.g., urology, obstetrics, and gynecology, in addition to infectious diseases, internal medicine, and intensive care units) of Hajar (48 isolates) and Kashani (32 isolates) Educational Hospitals during (21 April-22 October) 2016-2017. Additionally, using the statistical formula, the sample size was determined 80 by taking into account the maximum prevalence of $\mathrm{P}=\mathrm{Q}=0.50$, the error of 0.05 , and an accuracy of 0.11 . The inclusion criterion was Klebsiella isolates separated from patients with UTI 48-72 hours after hospitalization, and their primary urinary culture was negative at the time of referring to the hospital. In addition, the exclusion criterion encompassed a history of antibiotic usage. To isolate the samples, first, the information of the admitted patients including the hospitalization record and antibiotic usage within two weeks before the sampling, which was collected by using a questionnaire. The isolates were first cultured on blood Agar and eosin methylene blue (Himedia, India) and then the bacteria were identified employing biochemical differential tests such as the triple sugar iron, Simmons Citrate, methyl red, Voges-Proskauer, indole, mobility, oxidase, and catalase (Himedia, India). Following identifying and confirming the phenotypes of the Klebsiella bacterium (pneumonia and oxytoca), they were inoculated from the colony of the purified bacteria to $1.5 \mu \mathrm{M}$ microtubes containing $700 \mu \mathrm{L}$ of the sterile tryptic soy broth (Himedia, India) and incubated overnight at $37^{\circ} \mathrm{C}$. After the growth of the bacteria in the medium, $300 \mu \mathrm{L}$ of sterilized glycerol $(15 \%$ concentration) was added to medium and the bacteria were stored in a freezer at $-70^{\circ} \mathrm{C}$ to prevent the death of the bacteria in a long time. Further, the criteria proposed by the Clinical and Laboratory Standard Institute were used (17) in order to identify the Klebsiella strains. For this purpose, first, a suspension of the pure culture of the bacteria, equivalent to $0.5 \mathrm{McF}$ arland standard $(1.5 \times$ $10^{8} \mathrm{CFU} / \mathrm{mL}$ ), was prepared and then cultivated on the Mueller-Hinton agar (Merck, Germany). Next, the culture media were incubated at $37^{\circ} \mathrm{C}$ for $18-24$ hours. The antibiotic susceptibility of the bacteria was determined by disc diffusion method (Kirby-Bauer method) for two antibiotics, namely, meropenem and imipenem $(10 \mu \mathrm{g}$ each, MAST, England). After incubation, the diameter of the inhibition zone was measured and interpreted according to the standard antibiogram. Furthermore, the resistance of the strains to meropenem and imipenem was identified by determining the MIC using the E-test Liofilcheme (Italy) with a concentration range of 0.002-32 $\mu \mathrm{g} / \mathrm{mL}$. Moreover, the bacterial suspension, equal to 0.5 McFarland turbidity, was prepared from fresh and pure bacterial culture and cultivated on the Mueller-Hinton agar. Then, the strip was placed on the medium and incubated at $3{ }^{\circ} \mathrm{C}$ for $16-18$ hours (18-21).

\section{Results}

The data related to the isolates from different wards of the hospital are presented in Tables 1 and 2. As shown, most samples were collected from the patients in the urology ward while the least samples were taken from the intensive care unit (ICU). A number of 34 (42.5\%) and 46 (57.5\%) out of 80 patients with UTI, within the five-month period of sampling, were males and females, respectively, showing a higher incidence of UTI in women. In addition, respecting the prevalence of antibiotic resistance in men and women, the number of meropenem- and imipenemresistant samples was higher in women $(n=16)$ compared 
Table 1. The antibiotic results for meropenem and imipenem and the number of Klebsiella pneumoniae isolates in different wards

\begin{tabular}{lcccc}
\hline Wards & $\begin{array}{c}\text { Klebsiella } \\
\text { pneumonia }(\mathbf{n})\end{array}$ & $\begin{array}{c}\text { Resistant } \\
(\mathbf{n})\end{array}$ & $\begin{array}{c}\text { Semisensitive } \\
(\mathbf{n})\end{array}$ & $\begin{array}{c}\text { Sensitive } \\
(\mathbf{n})\end{array}$ \\
\hline Urology & 22 & 8 & 4 & 10 \\
$\begin{array}{l}\text { Obstetrics and } \\
\text { gynecology }\end{array}$ & 14 & 4 & 2 & 8 \\
Infectious diseases & 20 & 11 & 1 & 8 \\
Internal medicine & 10 & 0 & 0 & 10 \\
Intensive care unit & 8 & 0 & 2 & 6 \\
\hline
\end{tabular}

Table 2. The antibiotic results for both meropenem and imipenem antibiotics and the number of Klebsiella oxytoca isolates in different wards

\begin{tabular}{lcccc}
\hline Wards & $\begin{array}{c}\text { Klebsiella } \\
\text { oxytoca }(\mathbf{n})\end{array}$ & $\begin{array}{c}\text { Resistant } \\
(\mathbf{n})\end{array}$ & $\begin{array}{c}\text { Semisensitive } \\
(\mathbf{n})\end{array}$ & $\begin{array}{c}\text { Sensitive } \\
(\mathbf{n})\end{array}$ \\
\hline Urology & 2 & 1 & 0 & 1 \\
$\begin{array}{l}\text { Obstetrics and } \\
\text { gynecology }\end{array}$ & 1 & 0 & 1 & 0 \\
Infectious diseases & 2 & 1 & 0 & 1 \\
Internal medicine & 1 & 0 & 0 & 1 \\
Intensive care unit & 0 & 0 & 0 & 0 \\
\hline
\end{tabular}

to men $(\mathrm{n}=8)$, indicating a significantly higher prevalence of resistance to these antibiotics in women than men (Table 3). Additionally, as regards the age, the patients were within the age range of 4-72 years old. Further, most cases with UTI were 28-45 years old and the prevalence of antibiotic resistance was higher in older patients (34-45 years) compared to the younger ones. A total of $30 \%$ (i.e., 24 isolates including 22 pneumonia and 2 oxytoca isolates) were resistant to meropenem and imipenem based on the disc diffusion results (Tables 1 and 2). Furthermore, the E-test strips were read from the top to the bottom of the

Table 3. The antibiogram results of meropenem and imipenem in men and women

\begin{tabular}{lcc}
\hline & Totals samples & Resistant \\
\hline Men & 34 & 8 \\
Women & 46 & 16 \\
\hline
\end{tabular}

Table 4. The MIC of imipenem and meropenem antibiotics in Klebsiella pneumoniae isolates

\begin{tabular}{lccc}
\hline Antibiotic & $\begin{array}{c}\text { Resistant (n) } \\
\mathbf{M I C} \geq \mathbf{4}\end{array}$ & $\begin{array}{c}\text { Semisensitive (n) } \mathbf{1} \leq \\
\mathbf{M I C}<\mathbf{4}\end{array}$ & $\begin{array}{c}\text { Sensitive (n) } \\
\mathbf{M I C}<\mathbf{1}\end{array}$ \\
\hline Meropenem & 22 & 17 & 35 \\
Imipenem & 22 & 17 & 35 \\
\hline
\end{tabular}

Table 5. The MIC of imipenem and meropenem antibiotics in Klebsiella oxytoca isolates

\begin{tabular}{lccc}
\hline Antibiotic & $\begin{array}{c}\text { Resistant }(\mathbf{n}) \\
\mathrm{MIC} \geq \mathbf{4}\end{array}$ & $\begin{array}{c}\text { Semisensitive (n) } \\
\mathrm{MIC}<\mathbf{4}\end{array}$ & $\begin{array}{c}\text { Sensitive (n) } \\
\mathrm{MIC}<\mathbf{1}\end{array}$ \\
\hline Meropenem & 2 & 1 & 3 \\
Imipenem & 2 & 1 & 3 \\
\hline
\end{tabular}

strip (i.e., high to low concentrations of the antibiotics) after 18 hours of incubation. The antibiotic penetration from the strip into the medium containing agar caused an oval zone. Moreover, the MIC was decided to be the point where the oval zone crossed the strip $(21,22)$.

Based on the MICs, 24 (30\%) isolates were resistant to meropenem and imipenem with a MIC of $\geq 4 \mu \mathrm{g} / \mathrm{mL}$ whereas 18 isolates had a $\mathrm{MIC}$ of $1 \leq \mathrm{MIC}<4$ and were found to be semisensitive. Tables 4 and 5 separately present the MIC results for the Klebsiella species (i.e., pneumoniae and oxytoca). Only 2 out of the 6 oxytoca isolates were resistant to meropenem and imipenem, indicating the resistance among other Klebsiella species. Additionally, it demonstrates that this resistance is limited to no pneumonia.

Regarding the number of meropenem and imipenemresistant, the majority of the isolates were separated from the patients in the infectious disease wards. None of the strains were isolated from the internal ward and intensive care unit had a MIC of $\geq 4 \mu \mathrm{g} / \mathrm{mL}$. Based on the results of the disc diffusion method and E-test, the number of resistant isolates was equal. These isolates were completely resistant to meropenem and imipenem, and their MICs were even greater than $8 \mu \mathrm{g} / \mathrm{mL}$, which confirmed the high resistance of these samples by the diffusion method. However, the number of these strains (18 isolates) was higher in semisensitive strains compared to the disc diffusion results (10 isolates), which was due to the higher accuracy and greater sensitivity of the E-test strip compared to the disc diffusion method regarding identifying semi-sensitive and resistant strains. The significance level for the results of the Fisher exact test was considered $P<0.001$, and the results were found to be significant with respect to the number of strains isolated in each ward and the resistance level. Figures $1 \mathrm{~A}$ and $1 \mathrm{~B}$ illustrate the E-test bar for morphine and imipenem strains.

\section{Discussion}

The most common cause of UTI is Escherichia coli, followed by Klebsiella and Staphylococcus saprophyticus species (23). The importance of Klebsiella, as a human pathogenic agent, is related to the development of nosocomial infections and UTIs (24). The release of drug resistance factors in gram-negative bacteria and Klebsiella leads to an increase in the resistance of these microorganisms to various antibiotics. Therefore, therapeutic procedures for the patients are elongated $(25,26)$. In the present study, 24 isolates were meropenem and imipenem resistant with $\mathrm{MIC} \geq 4$ while 18 isolates were found to be semisensitive with $1 \leq \mathrm{MIC}<4$, which is in line with the results of Nobari et al in Iran, where in an investigation regarding resistance to carbapenems in 180 Klebsiella isolates, they observed that 42 isolates were resistant to meropenem whereas 29 and 14 isolated were resistant to ertapenem 

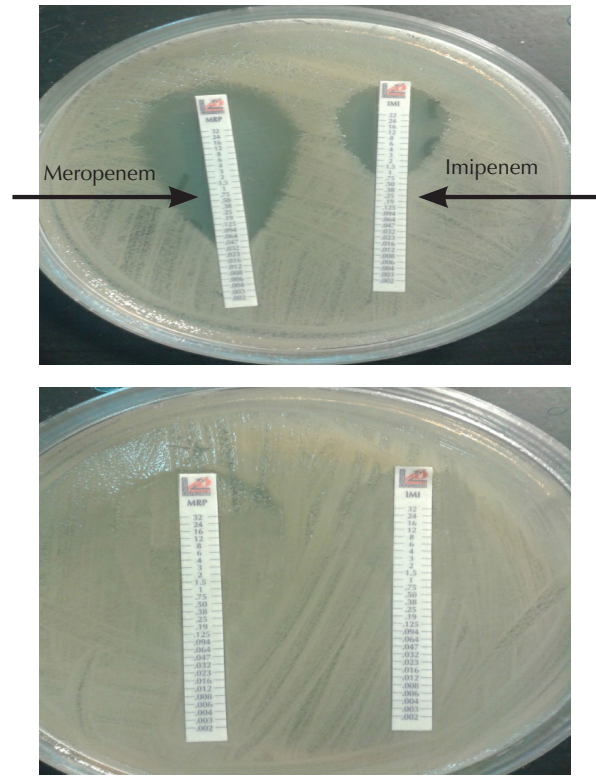

Figure 1. (A)The E-test strip of meropenem and imipenem-sensitive strain $(\mathrm{MIC}<1)$. (B) The E-test strip of meropenem and imipenem resistant strain.

and imipenem, respectively. In terms of the number of meropenem-resistant isolates in the total number of isolates, their results are consistent with the findings of the current study. The low number of imipenem-resistant strains compared to meropenem and ertapenem resistant ones in the study by Nobari et al may be due to several reasons such as the rate of treatment with imipenem in the study setting, the antibiotic susceptibility pattern of the studied samples, being inpatient or outpatient, and the like (27). In addition, in another study by Kanchanadevi et al in India, 25 out of 76 E. coli isolates, 2 out of 9 Klebsiella isolates, and 42 out of 60 Pseudomonas isolates were found to be resistant to imipenem based on the MIC results by using E-test, which is approximately consistent with the results of the current study with respect to the number of imipenem-resistant Klebsiella isolates per the total number of the isolates, indicating a lower prevalence of carbapenem resistance in Klebsiella and E. coli compared to Pseudomonas (28). Further, based on the results of previous studies, antibiotic resistance in Iran has been risen due to the excessive and over the counter use of antibiotics, as well as the acquisition of antibiotic resistance factors such as resistance genes spread through various methods including conjugation in the Klebsiella strains. The existence of this resistance was observed in the current study, which confirms this argument $(29,30)$. Furthermore, in a study by Tawfik et al, 87 inpatients in the ICU were investigated, 46 of whom were colonized with different bacterial agents. All of these bacteria were examined for resistance to carbapenems, the results of which demonstrated that 20 (43.5\%) Pseudomonas aeruginosa, 11 (23.9\%) Acinetobacter baumannii, 3 (6.5\%) E. coli, 2 (4.3\%) K. pneumoniae, and $1(2.2 \%)$ Enterobacter were resistant to carbapenems, which contradicts the results of the present study with respect to resistant Klebsiella isolates from the patients in the ICU. In this study, a total of 8 isolates were obtained from the patients in the ICU, none of which were resistant to meropenem and imipenem (31). Based on the findings and statistics, the prevalence of antibiotic-, meropenem-, and imipenem-resistant strains varies in different parts of the world. The results of the present study represented that Klebsiella strains isolated from patients with UTIs who were admitted to the wards of the hospital were significantly resistant to meropenem and imipenem, which indicates the colonization rate of Klebsiella strains in the hospital environment and higher sensitivity of these patients to obtain these organisms. The extensive and over-thecounter use of third-generation cephalosporins causes this resistance. If antibiotic resistance continues, antibiotics are likely to be effective in treating the infections resulted from these microorganisms. Moreover, the causes of low resistance to imipenem and meropenem compared to other antibiotics in various studies, as well as the current study, include low use of these antibiotics among the treatment teams and the lack of its use in outpatients since they are among the antibiotics used in hospitalized patients only with the physician' prescription, and the lower incidence of carbapnemase enzymes in the Enterobacteriaceae family compared to other bacteria (32-35). In a study conducted by Validi et al in Iran, of the 75 Klebsiella isolates obtained from the stool, blood, urine, phlegm, and wound, the least antibiotic resistance was observed with respect to meropenem and imipenem, and only one sample was resistant to these two antibiotics, which is not consistent with the results of the present study regarding the prevalence of resistance of these antibiotics. This can be due to the awareness of individuals in a region about the consequences of over-the-counter antibiotic use which makes the users predisposed to acquiring resistance, as well as the different prevalence of the genes responsible for this resistance in different regions (36). In this study, the most samples of UTI at the time of the specific sampling were from females, which indicates the higher incidence of the infection in women. This statistically corroborates with the findings of the other studies conducted in different parts of the world. The prevalence is more common in women compared to men, with almost half of the world's women experiencing this infection at least once in their lifetime (37). The proximity of the genitalia to the urethra (38), pregnancy, and sexual activity are the causes of the high prevalence of UTI in women. Additionally, many cases of UTI are treated as gastrointestinal or even respiratory infections, which increases the risk of developing pyelonephritis, urinary stones, preterm labor, and fetal death (39). In terms of age, the incidence of UTI increases with increasing age in both genders. However, the acquisition of this infection is lower in young men within the same duration, which matches the results of the current study where the number of UTI cases was lower 
in the people with lower ages (40). In addition, regarding the prevalence of antibiotic resistance in men and women, in the present study, the resistance to meropenem and imipenem was higher in women compared to men and in the middle-aged and older women compared to younger ones. Further, Doosti et al in their study on 120 samples of UTI found a comparatively higher prevalence of antibiotic resistance in women than men and in people aged 27 39 years and older compared to the younger ones (41). Furthermore, Mohammadi et al observed that resistance to antibiotics and beta-lactams was higher in women compared to men among the hospitalized patients (42), which is in line with the results of the current study with respect to the prevalence of resistance in women and men.

\section{Conclusion}

In general, based on the findings of this study, Klebsiella is the cause of UTI and resistant to meropenem and imipenem, which is consistent with the findings of other similar studies. Accordingly, physicians' awareness of resistance to antibiotics and bacteriological agents in every region is of great importance. Therefore, controlling the increase of this resistance is needed to prevent the overthe-counter use of antibiotics. Furthermore, pharmacies should not be allowed to sell the antibiotics without a doctor's prescription. As a result, future researchers are recommended to implement further studies on genes and enzymes that contribute to resistance to carbapenems and use faster diagnostic methods such as polymerase chain reaction in subsequent studies in order to prevent the increased therapeutic costs and the spread of this resistance.

Conflict of interests

None.

Ethical considerations

The study protocol obtained the ethics code IR.SKUMS. REC.1394.282 from Shahrekord University of Medical Sciences.

\section{Acknowledgments}

This study was a research project approved by Shahrekord University of Medical Sciences with the approval number of 2057 on 6 March 2016. We are grateful to the Vice-chancellor of the Research and Postgraduate Education of Shahrekord University of Medical Sciences, as well as all the people who helped us collect the samples and perform the present study.

\section{References}

1. Khan SA, Feroz F, Noor R. Study of extended-spectrum betalactamase-producing bacteria from urinary tract infections in Bangladesh. Tzu Chi Med J. 2013;25(1):39-42. doi: 10.1016/j. tcmj.2013.01.008.

2. Paterson DL, Bonomo RA. Extended-spectrum beta-lactamases: a clinical update. Clin Microbiol Rev. 2005;18(4):657-86. doi: 10.1128/cmr.18.4.657-686.2005.

3. Essack SY. Treatment options for extended-spectrum betalactamase-producers. FEMS Microbiol Lett. 2000;190(2):1814. doi: 10.1111/j.1574-6968.2000.tb09283.x.

4. Kil KS, Darouiche RO, Hull RA, Mansouri MD, Musher DM. Identification of a Klebsiella pneumoniae strain associated with nosocomial urinary tract infection. J Clin Microbiol. 1997;35(9):2370-4.

5. Daikos GL, Kosmidis C, Tassios PT, Petrikkos G, Vasilakopoulou A, Psychogiou M, et al. Enterobacteriaceae bloodstream infections: presence of integrons, risk factors, and outcome. Antimicrob Agents Chemother. 2007;51(7):2366-72. doi: 10.1128/aac.00044-07.

6. Green VL, Verma A, Owens RJ, Phillips SE, Carr SB. Structure of New Delhi metallo-beta-lactamase 1 (NDM-1). Acta Crystallogr Sect F Struct Biol Cryst Commun. 2011;67(Pt 10):1160-4. doi: 10.1107/s1744309111029654.

7. Fernandez A, Pereira MJ, Suarez JM, Poza M, Trevino $M$, Villalon $\mathrm{P}$, et al. Emergence in Spain of a multidrugresistant Enterobacter cloacae clinical isolate producing SFO-1 extended-spectrum beta-lactamase. J Clin Microbiol. 2011;49(3):822-8. doi: 10.1128/jcm.01872-10.

8. Mirsalehian A, Akbari Nakhjavani F, Bahador A, Jabal Ameli F, Bigverdi R, Goli HR. Prevalence of MBL-producing Pseudomonas aeruginosa isolated from burn patients. Tehran University Medical Journal. 2011;68(10):563-9.

9. Thomson KS. Extended-spectrum-beta-lactamase, AmpC, and Carbapenemase issues. J Clin Microbiol. 2010;48(4):1019-25. doi: 10.1128/jcm.00219-10.

10. Meletis G, Tzampaz E, Protonotariou E, Sofianou D. Emergence of Klebsiella pneumoniae carrying bla(VIM) and bla(KPC) genes. Hippokratia. 2010;14(2):139-40.

11. Sattar H, Toleman M, Nahid F, Zahra R. Co-existence of blaNDM-1 and blaKPC-2 in clinical isolates of Klebsiella pneumoniae from Pakistan. J Chemother. 2016;28(4):346-9. doi: $\quad 10.1179 / 1973947814 y .0000000223$.

12. Cagnacci S, Gualco L, Roveta S, Mannelli S, Borgianni L, Docquier JD, et al. Bloodstream infections caused by multidrug-resistant Klebsiella pneumoniae producing the carbapenem-hydrolysing VIM-1 metallo-beta-lactamase: first Italian outbreak. J Antimicrob Chemother. 2008;61(2):296300. doi: 10.1093/jac/dkm471.

13. Pitout JD, Nordmann P, Poirel L. Carbapenemase-producing Klebsiella pneumoniae, a key pathogen set for global nosocomial dominance. Antimicrob Agents Chemother. 2015;59(10):5873-84. doi: 10.1128/aac.01019-15.

14. Chen LF, Anderson DJ, Paterson DL. Overview of the epidemiology and the threat of Klebsiella pneumoniae carbapenemases (KPC) resistance. Infect Drug Resist. 2012;5:133-41. doi: 10.2147/idr.s26613.

15. Shields RK, Nguyen MH, Press EG, Chen L, Kreiswirth BN, Clancy CJ. In vitro selection of meropenem resistance among ceftazidime-avibactam-resistant, meropenemsusceptible Klebsiella pneumoniae isolates with variant KPC-3 carbapenemases. Antimicrob Agents Chemother. 2017;61(5). doi: 10.1128/aac.00079-17.

16. Kim YK, Pai H, Lee HJ, Park SE, Choi EH, Kim J, et al. Bloodstream infections by extended-spectrum beta-lactamaseproducing Escherichia coli and Klebsiella pneumoniae in children: epidemiology and clinical outcome. Antimicrob Agents Chemother. 2002;46(5):1481-91.

17. Wayne PA. Clinical and Laboratory Standards Institute: Performance standards for antimicrobial susceptibility testing: 20th informational supplement. CLSI; 2010.

18. Dong F, Zhang Y, Yao K, Lu J, Guo L, Lyu S, et al. Epidemiology of carbapenem-resistant Klebsiella pneumoniae bloodstream infections in a Chinese children's hospital: predominance of New Delhi metallo-beta-lactamase-1. Microb Drug Resist. 2018;24(2):154-60. doi: 10.1089/mdr.2017.0031.

19. Agrawal P, Ghosh AN, Kumar S, Basu B, Kapila K. Prevalence of extended-spectrum beta-lactamases among Escherichia coli and Klebsiella pneumoniae isolates in a tertiary care hospital. Indian J Pathol Microbiol. 2008;51(1):139-42.

20. Alberti MO, Hindler JA, Humphries RM. Performance of Etest 
for antimicrobial susceptibility testing of Abiotrophia defectiva and Granulicatella species. J Clin Microbiol. 2016;54(8):21946. doi: 10.1128/jcm.00822-16.

21. Direkel S, Uzunoglu E, Uzalp C, Findik E, Tontak S, Ahmadli C. Determination of Piperacillin/tazobactam and ticarcillin/ clavulanate susceptibilities in Pseudomonas aeruginosa isolates in hospitalised patients by E-test gradient method and comparison of results with disk diffusion tests. Clin Microbiol. 2017;6(1):1-4. doi: 10.4172/2327-5073.1000273.

22. Brown DF, Brown L. Evaluation of the E test, a novel method of quantifying antimicrobial activity. J Antimicrob Chemother. 1991;27(2):185-90.

23. Talan DA, Naber KG, Palou J, Elkharrat D. Extended-release ciprofloxacin (Cipro XR) for treatment of urinary tract infections. Int J Antimicrob Agents. 2004;23 Suppl 1:S54-66. doi: $\quad$ 10.1016/j.ijantimicag.2003.12.005.

24. Podschun R, Ullmann U. Klebsiella spp. as nosocomial pathogens: epidemiology, taxonomy, typing methods, and pathogenicity factors. Clin Microbiol Rev. 1998;11(4):589603.

25. Blondeau JM, Vaughan D. A review of antimicrobial resistance in Canada. Can J Microbiol. 2000;46(10):867-77.

26. Sharma VK, Johnson N, Cizmas L, McDonald TJ, Kim H. A review of the influence of treatment strategies on antibiotic resistant bacteria and antibiotic resistance genes. Chemosphere. 2016;150:702-14. doi: 10.1016/j. chemosphere.2015.12.084.

27. Nobari S, Shahcheraghi F, Rahmati Ghezelgeh F, Valizadeh B. Molecular characterization of carbapenem-resistant strains of Klebsiella pneumoniae isolated from Iranian patients: first identification of blaKPC gene in Iran. Microb Drug Resist. 2014;20(4):285-93. doi: 10.1089/mdr.2013.0074.

28. Kanchanadevi P, Chandra Sekaran S. Importance of EDTA in the detection of metallo beta lactamase from imipenem resistant gram negative bacilli. Int J Curr Microbiol App Sci. 2016;5(11):702-6. doi: 10.20546/ijcmas.2016.511.081.

29. Latifpour M, Gholipour A, Damavandi MS. The study of antibiotic resistance of extended-spectrum beta-lactamaseproducing Klebsiella strains isolated from urinary tract infections in teaching hospitals in Shahrekord. Journal of Shahrekord University of Medical Sciences. 2016;18(1):4553.

30. Kiani-Abari P, Zamanzad B, Gholipour A, Noormohamaddi Z. Determination and prevalence of antibiotic resistance in multi-drug resistant Klebsiella pneumonia in patients referred to the educational hospitals of Shahrekord in 2013. Journal of Shahrekord University of Medical Sciences. 2015;17(3):1217.

31. Tawfik AF, Shibl AM, Aljohi MA, Altammami MA, AlAgamy MH. Distribution of Ambler class A, B and D betalactamases among Pseudomonas aeruginosa isolates. Burns.
2012;38(6):855-60. doi: 10.1016/j.burns.2012.01.005.

32. Sirot DL, Goldstein FW, Soussy CJ, Courtieu AL, Husson MO, Lemozy J, et al. Resistance to cefotaxime and seven other beta-lactams in members of the family Enterobacteriaceae: a 3-year survey in France. Antimicrob Agents Chemother. 1992;36(8):1677-81.

33. Laupland KB, Parkins MD, Church DL, Gregson DB, Louie TJ, Conly JM, et al. Population-based epidemiological study of infections caused by carbapenem-resistant Pseudomonas aeruginosa in the Calgary Health Region: importance of metallo-beta-lactamase (MBL)-producing strains. J Infect Dis. 2005;192(9):1606-12. doi: 10.1086/444469.

34. Lin KY, Lauderdale TL, Wang JT, Chang SC. Carbapenemresistant Pseudomonas aeruginosa in Taiwan: Prevalence, risk factors, and impact on outcome of infections. J Microbiol Immunol Infect. 2016;49(1):52-9. doi: 10.1016/j. jmii.2014.01.005.

35. Logan LK, Bonomo RA. Metallo-beta-Lactamase (MBL)Producing Enterobacteriaceae in United States Children. Open Forum Infect Dis. 2016;3(2):ofw090. doi: 10.1093/ofid/ ofw090.

36. Validi M, Soltan Dallal MM, Douraghi M, Fallah Mehrabadi J, Rahimi Foroushani A. Identification of Klebsiella pneumoniae carbapenemase-producing Klebsiella oxytoca in clinical isolates in Tehran Hospitals, Iran by chromogenic medium and molecular methods. Osong Public Health Res Perspect. 2016;7(5):301-6. doi: 10.1016/j.phrp.2016.08.006.

37. Salvatore S, Salvatore S, Cattoni E, Siesto G, Serati M, Sorice $\mathrm{P}$, et al. Urinary tract infections in women. Eur J Obstet Gynecol Reprod Biol. 2011;156(2):131-6. doi: 10.1016/j. ejogrb.2011.01.028.

38. Schaeffer AJ, Rajan N, Cao Q, Anderson BE, Pruden DL, Sensibar J, et al. Host pathogenesis in urinary tract infections. Int J Antimicrob Agents. 2001;17(4):245-51.

39. Esmaeili M. Antibiotics for causative microorganisms of urinary tract infections. Iran J Pediatr. 2005;15(2):165-73.

40. Qin X, Hu F, Wu S, Ye X, Zhu D, Zhang Y, et al. Comparison of adhesin genes and antimicrobial susceptibilities between uropathogenic and intestinal commensal Escherichia coli strains. PLoS One. 2013;8(4):e61169. doi: 10.1371/journal. pone.0061169.

41. Doosti A, Daruoshi M, Borza R, Pasand M. Antibiotic resistance and distribution of beta-lactamase resistance genes in Escherichia coli strains isolated from urinary tract infection in women and children in the city Farsan. Journal of Shahrekord University of Medical Sciences. 2016;17(6):53-61.

42. Mohammadi M, Ghasemi E, Mokhayeri H, Pournia Y, Boroun $\mathrm{H}$. Antimicrobial resistance patterns of $E$. coli detected from hospitalized urine culture samples. Asian J Biol Sci. 2010;3(4):195-201. doi: 10.3923/ajbs.2010.195.201. 